\title{
Desempenho de sistemas alagados no tratamento de águas residuárias do processamento dos frutos do cafeeiro
}

\author{
Ronaldo Fia', Antonio T. de Matos ${ }^{2}$, Maria E. L. R. de Q ueiroz ${ }^{3}$, \\ Paulo R. Cecon ${ }^{4} \&$ Fátima R. L. Fia ${ }^{1}$
}

\begin{abstract}
RESU MO
Nove sistemas alagados (SACESH s) foram construídos em escala piloto (1,5 m de comprimento, 0,5 de largura e $0,4 \mathrm{~m}$ de profundidade), cultivados com Typha sp. e Alternanthera phyloxeroides, e operados sob diferentes taxas de aplicação orgânica, no tratamento da água residuária do processamento dos frutos do cafeeiro (ARC). Três sistemas receberam ARC sem nenhum pré-tratamento, em três foi aplicada ARC com correção de $\mathrm{pH}$ e correção nutricional e nos três últimos os efluentes de filtros anaeróbios utilizados no tratamento da $\mathrm{ARC}$, que teve $\mathrm{opH}$ e o teor de nutrientes corrigidos. $\mathrm{O}$ s resultados mostraram que 0 aumento nas taxas de carga superficial dos constituintes da ARC no sistema proporcionou decréscimo na eficiência de remoção de matéria orgânica e compostos fenólicos, em todos os SACESHs avaliados. Os tempos de detenção hidráulica superiores a $100 \mathrm{~h}$ proporcionaram maiores eficiências na remoção de DQO (SAC ${ }_{1}, \mathrm{SACF}_{1}$ e $\mathrm{SAC}_{2}$ ) e DBO $\left(\mathrm{SAC}_{1}, \mathrm{SAC}^{*}{ }_{1}, \mathrm{SAC}_{2}\right.$ e $\mathrm{SAC}_{3}$ ) que os tempos de detenção hidráulica de aproximadamente $60 \mathrm{~h}$ aplicados nas fases iniciais do experimento. Entre os SACESH s, 0 $\mathrm{SAC}^{\mathrm{F}}{ }_{1}$, que recebeu a menor taxa de aplicação orgânica (1.500 kg ha-1 $\mathrm{d}^{-1}$ de DQO), apresentou desempenho mais satisfatório no que se refere à remoção de DBO $(63 \%)$, DQO $(85 \%)$ e compostos fenólicos $(65 \%)$.
\end{abstract}

Palavras-chave: sistemas alagados construídos, água residuária agroindustrial, café, compostos fenólicos, matéria orgânica

\section{Performance of constructed wetlands used in the treatment of wastewater of coffee processing}

\begin{abstract}
Nine constructed wetlands (SACESH s) were built on a pilot scale ( $1.5 \mathrm{~m}$ long, $0.5 \mathrm{w}$ ide and $0.4 \mathrm{~m}$ deep), grown with Typha sp. and Alternanthera phyloxeroides, and operated under different organic loads in the treatment of wastewater of coffee processing (ARC). Three systems received no ARC pre-treatment, three received $A R C$ with correction of $\mathrm{pH}$ and nutritional correction, and the last three received the effluent of filters used in the anaerobic treatment of ARC that had the $\mathrm{pH}$ and nutrient content corrected. The results showed that the increase in the rate of surface charge of the constituents of the ARC provided the decrease in efficiency of removal of all SACESH s evaluated. The hydraulic detention time of more than $100 \mathrm{~h}$ provided higher efficiencies in the removal of $\operatorname{COD}\left(\mathrm{SAC}^{*},{ }_{1}, \mathrm{SAC}^{\mathrm{F}}\right.$ and $\left.\mathrm{SAC}_{2}\right)$ and $\mathrm{BOD}\left(\mathrm{SAC}_{1}\right.$, $\mathrm{SAC}^{*}{ }_{1}, \mathrm{SAC}_{2}$ and $\mathrm{SAC}_{3}{ }_{3}$ ) than hydraulic detention times of approximately $60 \mathrm{~h}$ implemented in the early stages of the experiment. Among SACESH s the $\mathrm{SAC}_{1}{ }_{1}$, which received the lowest organic load $(1500 \mathrm{~kg}$ $\mathrm{ha}^{-1} \mathrm{~d}^{-1}$ of COD), showed a satisfactory performance with regard to the removal of BOD $(63 \%)$, COD $(85 \%)$ and phenolic compounds $(65 \%)$.
\end{abstract}

Key words: constructed wetlands, wastewater agro-industry, coffee, phenolic compounds, organic matter

${ }^{1}$ DEG/U FLA. CP 3037, Campus da U FLA. CEP 37.200-000, Lavras, M G. Fone: (35) 3829-1481. E-mail: ronaldofia@deg.ufla.br; fatimarlf@deg.ufla.br ${ }^{2}$ DEA/UFV. Av. PH Rolfs s/n, Campus da UFV. CEP 36.570-000, Viçosa, M G. Fone: (31) 3899-2729. E-mail: atmatos@ufv.br

${ }^{3}$ DQI/UFV. Av. PH Rolfs s/n, Campus da UFV. Fone: (31) 3899-2370. E-mail: meliana@ufv.br

${ }^{4}$ DET/UFV. Av. PH Rolfs s/n, Campus da UFV. Fone: (31) 3899-2398. E-mail: cecon@ufv.br 


\section{INTRODUÇÃO}

Atualmente, o Brasil é o maior produtor e exportador de grãos de café e o processamento via úmida dos frutos, diferentemente da produção de grãos via seca, é o que apresenta maior tendência na busca de qualidade no produto. Assim, tornam-se imprescindíveis estudos através dos quais se possa considerar o tratamento das grandes quantidades de águas residuárias ricas em materiais orgânicos altamente poluentes geradas por este processo.

Diversas pesquisas têm enfocado o tratamento dessas águas residuárias em sistemas anaeróbios os quais apresentam, como vantagem, o fato de requererem menor área (Dinsdale et al., 1996; Bruno \& Oliveira, 2008; Fia et al., 2010); entretanto, embora os processos anaeróbios de tratamento alcancem elevada remoção de matéria orgânica biodegradável, a baixo custo, em geral seus efluentes não atendem às exigências da legislação ambiental para lançamento em corpos receptores requerendo, nesses casos, pós-tratamento. Pesquisas têm ressaltado a eficácia de sistemas alagados construídos no tratamento de águas residuárias brutas e no pós-tratamento de efluentes de sistemas anaeróbios (Brasil et al., 2005; Mazzola et al., 2005; Matos et al., 2010). Porém, informações sobre a aplicabilidade desse sistema no tratamento da água residuária do processamento dos frutos do cafeeiro (ARC) ainda são escassas. Trabalho anterior indicou resultados positivos com a utilização de sistemas alagados naturais cultivados com Typha para tratamento da ARC (Brasil et al., 2003); entretanto, o lançamento de águas residuárias em áreas alagadas naturais não constitui tratamento e, sim, disposição inadequada do resíduo. O tratamento em ambientes alagados só é permitido no caso da construção e no uso de sistemas alagados projetados com impermeabilização dos leitos de tratamento, objetivando-se a proteção do ambiente.

No presente trabalho se teve, como objetivo, estudar o desempenho de sistemas alagados construídos, cultivados com as macrófitas Typha sp. e Alternanthera philoxeroides, no tratamento de ARC, quando submetidos a diferentes condições experimentais e à aplicação de diferentes cargas orgânicas.

\section{MATERIAL E MÉTODOS}

O experimento foi conduzido na Área de Pré-Processamento e Armazenamento de Produtos Agrícolas, do Departamento de Engenharia Agrícola, na Universidade Federal de Viçosa, Viçosa, MG. Para sua condução, foram construídos nove sistemas alagados de escoamento subsuperficial horizontal (SACESHs), em escala piloto, que se compuseram de caixas (0,4 m de altura x 0,5 m de largura x 1,5 m de comprimento) de madeira de pinus, impermeabilizadas por geomembrana de polietileno de alta densidade e posicionadas sobre o solo em declividade de $0,01 \mathrm{~m} \mathrm{~m}^{-1}$. Como meio suporte utilizou-se brita zero (diâmetro $\mathrm{D}_{60}=7,0 \mathrm{~mm}$ e volume de vazios inicial de 0,491 $\mathrm{m}^{3} \mathrm{~m}^{-3}$ ). Os SACESHs foram preenchidos com a brita até a altura de 0,35 m, deixando-se uma borda livre (não saturada) de 0,05 m, já que o nível d'água foi mantido em 0,30 m (Figura 1). Em cada SACESH foi plantada, nos primeiros $0,75 \mathrm{~m}$ do leito, a espécie alternantera (Alternanthera phyloxeroides) e, nos últimos $0,75 \mathrm{~m}$, a taboa (Typha sp.).

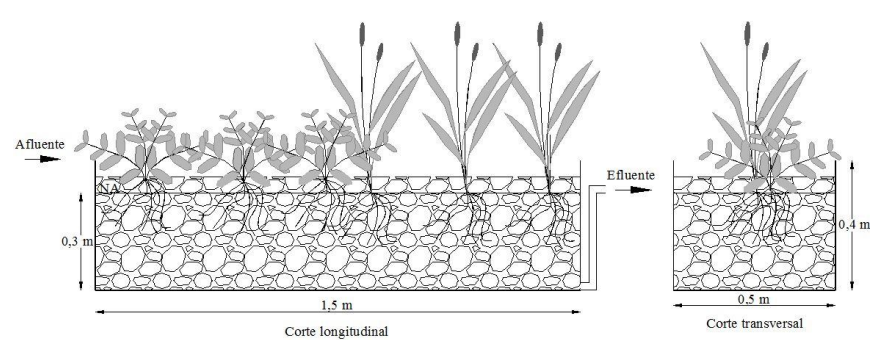

Figura 1. Esquema dos sistemas alagados construídos de escoamento subsuperficial horizontal (SACESHs)

A ARC utilizada no experimento proveio da Área de Préprocessamento e Armazenamento de Produtos Agrícolas da UFV e do Sítio Jatobá, situado a 12 km da cidade de Viçosa. As variações das características da ARC avaliadas em amostras coletadas durante todo o período experimental, estão apresentadas na Tabela 1 .

Tabela 1. Valores médios e desvio padrão das principais características da água residuária do processamento dos frutos do cafeeiro bruta utilizada nas três fases de condução do experimento

\begin{tabular}{lccc}
\hline \multirow{2}{*}{ Variáveis } & \multicolumn{3}{c}{ Fases } \\
\cline { 2 - 4 } & I (42 dias) & II (46 dias) & III (42 dias) \\
pH & $4,0 \pm 0,4$ & $3,7 \pm 0,2$ & $4,2 \pm 0,4$ \\
$\mathrm{DQO}\left(\mathrm{mg} \mathrm{L}^{-1}\right)$ & $12.241 \pm 8.100$ & $20.770 \pm 3.034$ & $10.151 \pm 2.191$ \\
$\mathrm{DBO}\left(\mathrm{mg} \mathrm{L}^{-1}\right)$ & $7.475 \pm 7.044$ & $10.186 \pm 2.350$ & $4.912 \pm 1.356$ \\
$\mathrm{~F}_{\mathrm{T}}\left(\mathrm{mg} \mathrm{L}^{-1}\right)$ & $75,8 \pm 29,4$ & $123,6 \pm 34,1$ & $79,5 \pm 9,4$ \\
\hline
\end{tabular}

pH - potencial hidrogeniônico; DQO - demanda química de oxigênio; DBO - demanda bioquímica de oxigênio; e $F_{T}$ - compostos fenólicos totais

Por se tratar de um sistema em escala piloto e, por isso, haver a necessidade de se aplicar pequenas vazões aos sistemas, a diferenciação entre as cargas orgânicas aplicadas foi feita por meio de diluição da ARC em água do sistema de abastecimento. Tendo em vista os reduzidos valores de $\mathrm{pH}$ apresentados pela ARC, foi patente a necessidade de sua correção para valores próximos à neutralidade, favorecendo a degradação microbiana (Metcalf \& Eddy, 2003). Optou-se pela cal, por ser um produto de menor custo e por favorecer o póstratamento por disposição no solo, quando comparado aos compostos à base de sódio. A correção nutricional da ARC (DBO/N/P = 100/5/1) foi necessária para favorecer o tratamento biológico, como descrito por Metcalf \& Eddy (2003) e confirmado por Fia et al. (2007).

Após dois meses de implantação do sistema iniciou-se a aplicação da ARC, sendo o experimento conduzido em três fases.

$\mathrm{Na}$ fase I, considerada a fase de adaptação do sistema à ARC, aplicou-se ARC diluída aos SACESHs. Nesta fase, três SACESHs receberam ARC diluída, três receberam ARC diluída e com correção de $\mathrm{pH}$ (elevação do $\mathrm{pH}$ até próximo de 7,0 com cal hidratada) e nos três últimos SACESHs se aplicaram os efluentes de filtros anaeróbios descritos por Fia et al. (2009), os quais foram alimentados com ARC diluída e correção de $\mathrm{pH}$; 
já na fase II se aumentou a taxa de aplicação orgânica, sendo o sistema conduzido através de três vias:

1. A ARC foi lançada diretamente nos SACESHs, em três concentrações diferentes: 50,75 e $100 \% \mathrm{v} \mathrm{v} \mathrm{v}^{-1}$, sendo os três SACESHs denominados $\mathrm{SAC}_{1}, \mathrm{SAC}_{2}$ e $\mathrm{SAC}_{3}$, respectivamente.

2. AARC recebeu correção com cal hidratada $\left(\mathrm{Ca}(\mathrm{OH})_{2}\right)$ até $\mathrm{pH}$ aproximadamente 7,0 e correção nutricional (DBO/N/P = 100/5/1) com uréia e superfosfato simples e foi lançada em três concentrações: 50,75 e $100 \% \mathrm{v} \mathrm{v}^{-1}$, sendo os três SACESHs denominados $\mathrm{SAC}_{1}{ }_{1}, \mathrm{SAC}_{2}{ }_{2} \mathrm{eSAC}_{3}{ }_{3}$, respectivamente.

3. A ARC recebeu correção com cal hidratada até $\mathrm{pH}$ aproximadamente 7,0 e correção nutricional (DBO/N/P = 100/ 5/1) e foi aplicada em filtros anaeróbios, em três concentrações: 50,75 e $100 \% \mathrm{v} \mathrm{v}^{-1}$, sendo os efluentes posteriormente lançados nos SACESHs denominados, respectivamente, $\mathrm{SAC}_{1}^{\mathrm{F}}, \mathrm{SAC}_{2}^{\mathrm{F}}{\text { e } \mathrm{SAC}^{\mathrm{F}}}_{3}$, como pós-tratamento; por fim se procedeu, na fase III, da mesma forma que na fase II, com exceção do tempo de detenção hidráulica (TDH) que foi aumentado aproximadamente duas vezes. As principais características operacionais dos SACESHs estão apresentadas na Tabela 2 .

O sistema de tratamento foi monitorado durante 130 dias, com amostragem semanal dos afluentes e dos efluentes dos SACESHs, quantificando-se as seguintes variáveis: $\mathrm{pH}$, por potenciometria; DBO, pelo método iodométrico; DQO, pelo método do refluxo aberto (APHA, 2005), além da concentração de compostos fenólicos totais $\left(\mathrm{F}_{\mathrm{T}}\right)$ (Folin \& Ciocalteu, 1927).

Tais variáveis foram avaliadas estatisticamente. Na fase I, o experimento foi montado no Delineamento em Blocos Casualizados (DBC), com 3 tratamentos (SAC, SAC ${ }^{*}$ e SAC: com as mesmas cargas orgânicas) e 3 repetições; nas fases II e III, as amostragens foram tomadas como repetições, sendo o experimento montado no DBC com 9 tratamentos $\left(\mathrm{SAC}_{1}, \mathrm{SAC}_{2}\right.$, $\mathrm{SAC}_{3}, \mathrm{SAC}_{1}^{*}, \mathrm{SAC}_{2}^{*}, \mathrm{SAC}_{3}^{*}, \mathrm{SAC}_{1}^{\mathrm{F}}, \mathrm{SAC}_{2}{ }_{2}$ e $\mathrm{SAC}_{3}{ }_{3}$ ) e 5 repetições para cada fase. Os dados foram analisados por meio da análise de variância e as médias comparadas utilizando-se o teste de Tukey, adotando-se o nível de 10\% de probabilidade. As análises estatísticas foram feitas através do programa estatístico $\mathrm{SAEG}^{\circledR}$.

\section{RESULTADOS E DISCUSSÃO}

Nas fases I, II e III, os SACESHs operaram numa faixa de temperatura considerada psicrofílica para os micro-organismos. Para as respectivas fases, as temperaturas médias diárias do líquido foram iguais a 17,$6 ; 18,4$ e $20,4^{\circ} \mathrm{C}$, sendo os extremos mínimos e máximos registrados de 13,8 e $24,4^{\circ} \mathrm{C}$. Com relação à ocorrência de chuvas foi registrada, durante a fase experimental, apenas uma precipitação de $15,4 \mathrm{~mm}$ ocorrida no $121^{\circ}$ dia após o início da condução do experimento, a qual não foi capaz de influenciar os resultados experimentais.

Na Tabela 3 se encontra a caracterização dos afluentes e dos efluentes dos SACESHs, com relação às variáveis $\mathrm{pH}$, $\mathrm{DBO}$, DQO e $\mathrm{F}_{\mathrm{T}}$, durante as três fases de operação dos sistemas.

Verificou-se nos SACs, durante as três fases, elevação no pH efluente, o que pode estar associado à liberação de cátions de reação básica pela brita utilizada como material suporte. Durante a fase I os valores de $\mathrm{pH}$ efluente dos $\mathrm{SAC}^{*} \mathrm{~s}$ e $\mathrm{SAC}^{\mathrm{F}} \mathrm{s}$ oscilaram (Tabela 3) mas foram semelhantes aos valores afluentes e as médias não diferiram entre si $(\mathrm{p}>0,1)$; na fase II ocorreu redução acentuada nos valores de $\mathrm{pH}$ nos $\mathrm{SAC}^{*} \mathrm{~s}$, devido ao aumento da taxa de aplicação orgânica superficial; nos $\mathrm{SAC}^{\mathrm{F}} \mathrm{S}$ este efeito somente foi observado para o $\mathrm{SAC}_{3}^{\mathrm{F}}$, o qual recebeu a maior $\mathrm{TCO}_{\mathrm{A}}$, dentre todos os $\mathrm{SAC}^{\mathrm{F}} \mathrm{s}$. Os valores observados de $\mathrm{pH}$ estiveram dentro da faixa na qual são proporcionadas condições ideais para a sobrevivência das bactérias responsáveis pela degradação orgânica, que varia

Tabela 2. Características operacionais dos nove sistemas alagados construídos de escoamento subsuperficial horizontal (SACESH S)

\begin{tabular}{|c|c|c|c|c|}
\hline \multirow{2}{*}{ SACESHs } & \multirow{2}{*}{ Variáveis } & \multicolumn{3}{|c|}{ Fases } \\
\hline & & I & II & III \\
\hline $\mathrm{SAC}_{1}$ & $\begin{array}{l}\text { TDH (h) } \\
\operatorname{TCO}_{\mathrm{A}}\left(\mathrm{kg} \mathrm{ha}^{-1} \mathrm{~d}^{-1}\right)\end{array}$ & $\begin{array}{l}64,1 \pm 15,3^{(42)} \\
1.523 \pm 610^{(5)}\end{array}$ & $\begin{array}{c}58,9 \pm 8,9^{(42)} \\
4.184 \pm 521^{(5)}\end{array}$ & $\begin{array}{c}111,5 \pm 20,6^{(42)} \\
1.281 \pm 328^{(5)}\end{array}$ \\
\hline $\mathrm{SAC}_{2}$ & $\begin{array}{l}\text { TDH (h) } \\
\operatorname{TCO}_{\mathrm{A}}\left(\mathrm{kg} \mathrm{ha}^{-1} \mathrm{~d}^{-1}\right)\end{array}$ & $\begin{array}{l}59,3 \pm 10,9^{(42)} \\
1.732 \pm 541^{(5)}\end{array}$ & $\begin{array}{c}58,6 \pm 11,8^{(42)} \\
8.322 \pm 1.379^{(5)}\end{array}$ & $\begin{array}{c}107,4 \pm 20,3^{(42)} \\
2.139 \pm 775^{(5)}\end{array}$ \\
\hline $\mathrm{SAC}_{3}$ & $\begin{array}{l}\text { TDH (h) } \\
\operatorname{TCO}_{A}\left(k g h a^{-1} d^{-1}\right)\end{array}$ & $\begin{array}{l}58,0 \pm 10,2^{(42)} \\
1.668 \pm 538^{(5)}\end{array}$ & $\begin{array}{c}57,9 \pm 12,1^{(42)} \\
13.528 \pm 5.763^{(5)}\end{array}$ & $\begin{array}{c}98,6 \pm 19,8^{(42)} \\
3.083 \pm 1.228^{(5)}\end{array}$ \\
\hline $\mathrm{SAC}{ }_{1}$ & $\begin{array}{l}\text { TDH (h) } \\
\mathrm{TCO}_{\mathrm{A}}\left(\mathrm{kg} \mathrm{ha-1} \mathrm{d}^{-1}\right)\end{array}$ & $\begin{array}{l}58,2 \pm 10,6^{(42)} \\
1.417 \pm 645^{(5)}\end{array}$ & $\begin{array}{c}58,1 \pm 11,7^{(42)} \\
4.401 \pm 1.492^{(5)}\end{array}$ & $\begin{array}{c}110,6 \pm 28,3^{(42)} \\
1.418 \pm 335^{(5)}\end{array}$ \\
\hline $\mathrm{SAC}_{2}$ & $\begin{array}{l}\text { TDH (h) } \\
\mathrm{TCO}_{\mathrm{A}}\left(\mathrm{kg} \mathrm{ha-1} \mathrm{d}^{-1}\right)\end{array}$ & $\begin{array}{c}55,3 \pm 10,0^{(42)} \\
1.810 \pm 1.164^{(5)}\end{array}$ & $\begin{array}{c}59,0 \pm 15,8^{(42)} \\
6.029 \pm 1.602^{(5)}\end{array}$ & $\begin{array}{c}94,5 \pm 22,4^{(42)} \\
2.707 \pm 1.089^{(5)}\end{array}$ \\
\hline $\mathrm{SAC}_{3}$ & $\begin{array}{l}\text { TDH (h) } \\
\mathrm{TCO}_{\mathrm{A}}\left(\mathrm{kg} \mathrm{ha-1} \mathrm{d}^{-1}\right)\end{array}$ & $\begin{array}{c}55,1 \pm 9,6^{(42)} \\
1.633 \pm 877^{(5)}\end{array}$ & $\begin{array}{c}63,7 \pm 15,9^{(42)} \\
10.264 \pm 4.516^{(5)}\end{array}$ & $\begin{array}{l}104,8 \pm 31,1^{(42)} \\
3.476 \pm 2.094^{(5)}\end{array}$ \\
\hline $\operatorname{SAC}_{1}{ }_{1}$ & $\begin{array}{l}\text { TDH (h) } \\
\mathrm{TCO}_{\mathrm{A}}\left(\mathrm{kg} \mathrm{ha}{ }^{-1} \mathrm{~d}^{-1}\right)\end{array}$ & $\begin{array}{c}58,7 \pm 7,4^{(42)} \\
1.058 \pm 586^{(5)}\end{array}$ & $\begin{array}{c}54,4 \pm 13,7^{(42)} \\
3.597 \pm 1.165^{(5)}\end{array}$ & $\begin{array}{c}111,8 \pm 27,0^{(42)} \\
1.507 \pm 213^{(5)}\end{array}$ \\
\hline $\mathrm{SAC}_{2}$ & $\begin{array}{l}\text { TDH (h) } \\
\mathrm{TCO}_{\mathrm{A}}\left(\mathrm{kg} \mathrm{ha} \mathrm{ha}^{-1} \mathrm{~d}^{-1}\right)\end{array}$ & $\begin{array}{l}57,6 \pm 5,0^{(42)} \\
805 \pm 280^{(5)}\end{array}$ & $\begin{array}{c}61,0 \pm 13,4^{(42)} \\
6.006 \pm 1.635^{(5)}\end{array}$ & $\begin{array}{l}126,7 \pm 24,1^{(42)} \\
2.2579 \pm 793^{(5)}\end{array}$ \\
\hline $\mathrm{SAC}_{3}$ & $\begin{array}{l}\text { TDH (h) } \\
\mathrm{TCO}_{\mathrm{A}}\left(\mathrm{kg} \mathrm{ha} \mathrm{h}^{-1} \mathrm{~d}^{-1}\right)\end{array}$ & $\begin{array}{l}58,6 \pm 9,9^{(42)} \\
798 \pm 409^{(5)}\end{array}$ & $\begin{array}{c}59,5 \pm 14,2^{(42)} \\
9.092 \pm 4.559^{(5)}\end{array}$ & $\begin{array}{l}114,7 \pm 22,5^{(42)} \\
3.043 \pm 1.076^{(5)}\end{array}$ \\
\hline
\end{tabular}

(a) Entre parênteses o número de amostragens considerado no cálculo da média

TDH - tempo de detenção hidráulica; TCOA - taxa de aplicação orgânica superficial tomada com base na concentração da DQO 
Tabela 3. Valores médios e desvio padrão de potencial hidrogeniônico (pH), demanda química de oxigênio (DQ 0 ), demanda bioquímica de oxigênio (DBO) e compostos fenólicos totais $\left(F_{T}\right)$ afluente e efluente dos sistemas alagados construídos de escoamento subsuperficial horizontal (SACESH s), em cada fase operacional

\begin{tabular}{|c|c|c|c|c|c|c|c|}
\hline \multirow{2}{*}{ Variáveis } & \multirow{2}{*}{ SACESHS } & \multicolumn{2}{|c|}{ Fase I } & \multicolumn{2}{|c|}{ Fase II } & \multicolumn{2}{|c|}{ Fase III } \\
\hline & & Afluente & Efluente & Afluente & Efluente & Afluente & Efluente \\
\hline \multirow{5}{*}{$\mathrm{pH}$} & $\mathrm{SAC}_{1}$ & $4,32 \pm 0,85$ & $5,99 \pm 0,26$ & $3,52 \pm 0,12$ & $4,66 \pm 0,12$ & $4,47 \pm 0,20$ & $4,74 \pm 0,19$ \\
\hline & $\mathrm{SAC}_{2}$ & $4,44 \pm 0,94$ & $5,73 \pm 0,14$ & $3,55 \pm 0,12$ & $4,34 \pm 0,31$ & $4,38 \pm 0,43$ & $4,59 \pm 0,13$ \\
\hline & $\mathrm{SAC}_{3}$ & $4,47 \pm 0,79$ & $5,77 \pm 0,23$ & $3,56 \pm 0,13$ & $4,31 \pm 0,48$ & $4,30 \pm 0,35$ & $4,36 \pm 0,11$ \\
\hline & $\begin{array}{l}\text { SAC* }_{1} \\
\text { SAC* }{ }_{2} \\
\text { SAC* }_{3} \\
\end{array}$ & $\begin{array}{l}6,56 \pm 0,37 \\
6,56 \pm 0,36 \\
6,57 \pm 0,29 \\
\end{array}$ & $\begin{array}{l}6,66 \pm 0,69 \\
6,71 \pm 0,51 \\
6,66 \pm 0,60 \\
\end{array}$ & $\begin{array}{l}6,24 \pm 0,57 \\
6,53 \pm 0,85 \\
6,26 \pm 0,98 \\
\end{array}$ & $\begin{array}{l}6,39 \pm 0,65 \\
6,18 \pm 0,32 \\
6,07 \pm 0,28 \\
\end{array}$ & $\begin{array}{l}7,44 \pm 0,72 \\
7,83 \pm 0,15 \\
7,84 \pm 0,31 \\
\end{array}$ & $\begin{array}{l}7,83 \pm 0,17 \\
7,39 \pm 0,46 \\
6,93 \pm 0,19 \\
\end{array}$ \\
\hline & $\begin{array}{l}\operatorname{SAC}_{1} \\
\operatorname{SAC}_{2} \\
\operatorname{SAC}_{3} \\
\end{array}$ & $\begin{array}{l}6,83 \pm 0,52 \\
6,92 \pm 0,39 \\
6,85 \pm 0,52 \\
\end{array}$ & $\begin{array}{l}7,21 \pm 0,38 \\
7,25 \pm 0,31 \\
7,36 \pm 0,59 \\
\end{array}$ & $\begin{array}{l}6,55 \pm 0,38 \\
6,21 \pm 0,30 \\
6,08 \pm 0,27 \\
\end{array}$ & $\begin{array}{l}7,39 \pm 0,18 \\
6,95 \pm 0,19 \\
6,49 \pm 0,51 \\
\end{array}$ & $\begin{array}{l}7,67 \pm 0,10 \\
7,06 \pm 0,09 \\
6,99 \pm 0,72 \\
\end{array}$ & $\begin{array}{l}7,71 \pm 0,26 \\
7,84 \pm 0,11 \\
7,50 \pm 0,23 \\
\end{array}$ \\
\hline \multirow{3}{*}{$\begin{array}{c}\mathrm{DQO} \\
\left(\mathrm{mg} \mathrm{L}^{-1}\right)\end{array}$} & $\begin{array}{l}\mathrm{SAC}_{1} \\
\mathrm{SAC}_{2} \\
\mathrm{SAC}_{3} \\
\end{array}$ & $\begin{array}{l}2.275 \pm 775 \\
2.576 \pm 846 \\
2.420 \pm 967 \\
\end{array}$ & $\begin{array}{l}1.105 \pm 308 \\
1.272 \pm 241 \\
1.280 \pm 326 \\
\end{array}$ & $\begin{array}{c}6.837 \pm 969 \\
11.508 \pm 2.246 \\
19.084 \pm 8.091 \\
\end{array}$ & $\begin{array}{c}5.592 \pm 354 \\
9.443 \pm 1.723 \\
12.466 \pm 3.452 \\
\end{array}$ & $\begin{array}{c}3.539 \pm 548 \\
5.615 \pm 1.133 \\
7.771 \pm 1.590 \\
\end{array}$ & $\begin{array}{c}2.746 \pm 279 \\
4.435 \pm 934 \\
5.502 \pm 2.277 \\
\end{array}$ \\
\hline & $\begin{array}{l}\text { SAC* }_{1} \\
\text { SAC* }_{2} \\
\text { SAC* }_{3} \\
\end{array}$ & $\begin{array}{l}2.161 \pm 1.167 \\
2.317 \pm 1.184 \\
2.258 \pm 1.205\end{array}$ & $\begin{array}{l}1.521 \pm 506 \\
1.582 \pm 482 \\
1.572 \pm 754 \\
\end{array}$ & $\begin{array}{c}6.536 \pm 1.290 \\
11.544 \pm 2.846 \\
19.566 \pm 9.697\end{array}$ & $\begin{array}{c}6.017 \pm 1.432 \\
10.221 \pm 2.498 \\
17.143 \pm 9.093 \\
\end{array}$ & $\begin{array}{c}4.182 \pm 752 \\
6.886 \pm 588 \\
9.418 \pm 1.162 \\
\end{array}$ & $\begin{array}{l}2.343 \pm 1.259 \\
5.235 \pm 1.394 \\
7.040 \pm 2.026\end{array}$ \\
\hline & $\begin{array}{l}\operatorname{SAC}_{1}{ }_{1} \\
\operatorname{SAC}_{2} \\
\operatorname{SAC}_{3} \\
\end{array}$ & $\begin{array}{l}1.612 \pm 831 \\
1.285 \pm 394 \\
1.353 \pm 768 \\
\end{array}$ & $\begin{array}{l}748 \pm 409 \\
824 \pm 389 \\
736 \pm 487 \\
\end{array}$ & $\begin{array}{c}5.658 \pm 1.093 \\
9.792 \pm 2.162 \\
16.036 \pm 7.333 \\
\end{array}$ & $\begin{array}{c}1.881 \pm 398 \\
7.096 \pm 3.826 \\
14.274 \pm 10.021 \\
\end{array}$ & $\begin{array}{c}4.182 \pm 752 \\
6.886 \pm 588 \\
9.072 \pm 1.322 \\
\end{array}$ & $\begin{array}{c}587 \pm 323 \\
1.786 \pm 1.066 \\
5.940 \pm 2.789\end{array}$ \\
\hline \multirow{3}{*}{$\begin{array}{c}\mathrm{DBO} \\
\left(\mathrm{mg} \mathrm{L}^{-1}\right)\end{array}$} & $\begin{array}{l}\mathrm{SAC}_{1} \\
\mathrm{SAC}_{2} \\
\mathrm{SAC}_{3}\end{array}$ & $\begin{array}{l}905 \pm 399 \\
925 \pm 337 \\
853 \pm 280 \\
\end{array}$ & $\begin{array}{l}642 \pm 190 \\
746 \pm 225 \\
661 \pm 251 \\
\end{array}$ & $\begin{array}{c}2.011 \pm 825 \\
3.535 \pm 883 \\
4.441 \pm 1.365 \\
\end{array}$ & $\begin{array}{l}1.966 \pm 1.125 \\
2.957 \pm 1.784 \\
1.985 \pm 1.244\end{array}$ & $\begin{array}{c}1.698 \pm 884 \\
3.176 \pm 2.134 \\
3.348 \pm 1.941\end{array}$ & $\begin{array}{c}905 \pm 561 \\
1.879 \pm 1.152 \\
975 \pm 604\end{array}$ \\
\hline & $\begin{array}{l}\text { SAC* }_{1} \\
\text { SAC* }_{2} \\
\text { SAC* }_{3} \\
\end{array}$ & $\begin{array}{l}1.292 \pm 474 \\
1.401 \pm 590 \\
1.183 \pm 408 \\
\end{array}$ & $\begin{array}{l}935 \pm 287 \\
806 \pm 347 \\
966 \pm 406 \\
\end{array}$ & $\begin{array}{c}3.242 \pm 771 \\
4.178 \pm 781 \\
5.874 \pm 1.019 \\
\end{array}$ & $\begin{array}{c}2.327 \pm 605 \\
3.510 \pm 619 \\
3.798 \pm 1.553\end{array}$ & $\begin{array}{c}2.517 \pm 1.241 \\
3.250 \pm 972 \\
4.399 \pm 1.012\end{array}$ & $\begin{array}{l}1.175 \pm 993 \\
3.012 \pm 977 \\
3.583 \pm 923 \\
\end{array}$ \\
\hline & $\begin{array}{l}\operatorname{SAC}_{1} \\
\operatorname{SAC}_{2} \\
\operatorname{SAC}_{3} \\
\end{array}$ & $\begin{array}{c}1.036 \pm 502 \\
965 \pm 300 \\
722 \pm 366 \\
\end{array}$ & $\begin{array}{l}473 \pm 382 \\
537 \pm 317 \\
384 \pm 283 \\
\end{array}$ & $\begin{array}{c}3.184 \pm 717 \\
4.311 \pm 887 \\
6.181 \pm 1.457 \\
\end{array}$ & $\begin{array}{c}787 \pm 303 \\
3.556 \pm 1.744 \\
5.247 \pm 2.695 \\
\end{array}$ & $\begin{array}{c}1.336 \pm 1.105 \\
2.772 \pm 874 \\
4.884 \pm 1.103 \\
\end{array}$ & $\begin{array}{c}359 \pm 283 \\
1.355 \pm 1.144 \\
2.856 \pm 2.071 \\
\end{array}$ \\
\hline \multirow{3}{*}{$\begin{array}{c}\mathrm{F}_{\mathrm{T}} \\
\left(\mathrm{mg} \mathrm{L}^{-1}\right)\end{array}$} & $\begin{array}{l}\mathrm{SAC}_{1} \\
\mathrm{SAC}_{2} \\
\mathrm{SAC}_{3} \\
\end{array}$ & $\begin{array}{l}19,3 \pm 8,1 \\
22,9 \pm 7,9 \\
19,8 \pm 8,3\end{array}$ & $\begin{array}{l}10,0 \pm 6,8 \\
14,6 \pm 5,4 \\
13,7 \pm 6,5\end{array}$ & $\begin{array}{r}51,5 \pm 11,2 \\
79,0 \pm 13,1 \\
102,4 \pm 12,9 \\
\end{array}$ & $\begin{array}{c}45,6 \pm 5,1 \\
76,5 \pm 22,2 \\
99,0 \pm 8,0 \\
\end{array}$ & $\begin{array}{l}28,0 \pm 5,5 \\
47,2 \pm 5,3 \\
67,7 \pm 8,3 \\
\end{array}$ & $\begin{array}{l}36,3 \pm 6,0 \\
51,4 \pm 5,7 \\
64,4 \pm 7,6 \\
\end{array}$ \\
\hline & $\begin{array}{l}\text { SAC* }_{1} \\
\text { SAC* }_{2} \\
\text { SAC* }_{3} \\
\end{array}$ & $\begin{array}{l}14,7 \pm 2,2 \\
13,2 \pm 4,5 \\
12,2 \pm 3,8 \\
\end{array}$ & $\begin{array}{l}7,8 \pm 5,5 \\
9,8 \pm 4,9 \\
6,5 \pm 4,8 \\
\end{array}$ & $\begin{array}{l}30,1 \pm 26,6 \\
49,4 \pm 41,5 \\
65,0 \pm 42,1 \\
\end{array}$ & $\begin{array}{c}18,4 \pm 9,2 \\
33,0 \pm 18,3 \\
40,1 \pm 23,6 \\
\end{array}$ & $\begin{array}{c}6,8 \pm 4,2 \\
13,7 \pm 6,0 \\
19,4 \pm 8,4 \\
\end{array}$ & $\begin{array}{c}3,6 \pm 4,4 \\
5,9 \pm 4,3 \\
14,5 \pm 6,3 \\
\end{array}$ \\
\hline & $\begin{array}{l}\operatorname{SAC}_{1} \\
\operatorname{SAC}_{2} \\
\operatorname{SAC}_{3}\end{array}$ & $\begin{array}{c}10,4 \pm 3,6 \\
8,7 \pm 4,6 \\
7,0 \pm 2,9\end{array}$ & $\begin{array}{l}3,3 \pm 1,2 \\
4,3 \pm 2,5 \\
4,2 \pm 2,7\end{array}$ & $\begin{array}{l}13,0 \pm 3,4 \\
20,2 \pm 2,1 \\
28,3 \pm 6,5\end{array}$ & $\begin{array}{c}5,6 \pm 0,6 \\
14,1 \pm 3,0 \\
23,0 \pm 3,2\end{array}$ & $\begin{array}{l}29,1 \pm 20,8 \\
45,4 \pm 30,8 \\
64,3 \pm 44,7\end{array}$ & $\begin{array}{c}9,2 \pm 4,2 \\
15,9 \pm 7,5 \\
41,4 \pm 32,5\end{array}$ \\
\hline
\end{tabular}

Fase I: fase de adaptação em que os SACESHs receberam ARC diluída. Fase II: aumento da taxa de aplicação orgânica aos SACESHs em três diluições (50, 75 e 100\%). Fase III: aumento do TDH em aproximadamente duas vezes ao aplicado na fase II

de 6,0 a 9,5 (Metcalf \& Eddy, 2003), à exceção dos valores médios obtidos no afluente e efluentes dos SACs, que se mantiveram abaixo de 6,0, em todo o período experimental.

Obtiveram-se, durante a fase I, eficiências relativamente baixas na remoção de $\mathrm{DQO}$ e DBO, pois os sistemas estavam ainda em fase de adaptação (Tabela 4). Nesta fase, dentre os SACESHs avaliados, eficiências relativamente maiores de remoção de DBO foram obtidas nos $\mathrm{SAC}^{\mathrm{F}} \mathrm{s}(\mathrm{p}<0,1)$; acreditase que, por passar pelos filtros anaeróbios, a ARC lançada nos $\mathrm{SAC}^{\mathrm{F}} \mathrm{S}$ apresentava frações orgânicas de mais fácil degradação, com a quebra de fibras celulósicas, ligninas e compostos fenólicos. Além disso, o carreamento de biomassa dos filtros para os $\mathrm{SAC}^{\mathrm{F}} \mathrm{s}$ pode ter favorecido a formação do biofilme, que é de extrema importância para a remoção da matéria orgânica em sistemas alagados. Dentre os $\mathrm{SAC}^{\mathrm{F}} \mathrm{S}, \mathrm{o} \mathrm{SAC}^{\mathrm{F}}$, foi aquele que apresentou maiores eficiências na remoção de matéria orgânica em virtude das menores cargas orgânicas aplicadas, sendo estatisticamente diferente dos e $\mathrm{SAC}_{1}$ e $\mathrm{SAC}_{1}{ }_{1}(\mathrm{p}<0,1)$ quanto à remoção de DQO na fase III.

No início da fase II notou-se tendência de queda nas eficiências de remoção de $\mathrm{DBO}$ e $\mathrm{DQO}$, exceto para o $\mathrm{SAC}^{\mathrm{F}}$, provavelmente como consequência do aumento da $\mathrm{TCO}_{\mathrm{A}}$ aplicada; na fase III a maioria dos SACESHs apresentou recuperação da eficiência na remoção de matéria orgânica, fato atribuído à adaptação do sistema à ARC, ao aumento do TDH e também à presença dos nutrientes adicionados à ARC, a partir da fase II.

Vrhovsek et al. (1996) obtiveram eficiências de 89 e 92\%, respectivamente, na remoção de $\mathrm{DBO}$ e $\mathrm{DQO}$, no tratamento de águas residuárias da indústria de extração de pigmentos e compostos ativos de vegetais, quando utilizaram sistemas alagados construídos, operando em série e precedidos por um sedimentador. Nessas avaliações os autores aplicaram 1.180 $\mathrm{kg} \mathrm{ha}^{-1} \mathrm{~d}^{-1}$ de DQO nos sistemas alagados construídos. 
Tabela 4. Valores das eficiências médias de remoção (\%) e desvio padrão de matéria orgânica (DBO - demanda bioquímica de oxigênio e DQ 0 - demanda química de oxigênio) e compostos fenólicos $\left(F_{T}\right)$ nos sistemas alagados construídos de escoamento subsuperficial horizontal (SACESH s), em cada fase operacional

\begin{tabular}{|c|c|c|c|c|c|c|c|c|c|}
\hline \multirow{2}{*}{ SACESHS } & \multicolumn{3}{|c|}{ Fase I } & \multicolumn{3}{|c|}{ Fase II } & \multicolumn{3}{|c|}{ Fase III } \\
\hline & DQO & DBO & $\mathbf{F}_{\mathrm{T}}$ & DQO & DBO & $\mathbf{F}_{\mathrm{T}}$ & DQO & DBO & $\mathbf{F}_{\mathrm{T}}$ \\
\hline $\mathrm{SAC}_{1}$ & $47 \pm 18$ & $22 \pm 27$ & $43 \pm 41$ & $17 \pm 7$ & $5 \pm 35$ & $10 \pm 12$ & $21 \pm 12$ & $47 \pm 9$ & $-32 \pm 24$ \\
\hline $\mathrm{SAC}_{2}$ & $47 \pm 17$ & $16 \pm 21$ & $30 \pm 30$ & $18 \pm 5$ & $22 \pm 39$ & $4 \pm 14$ & $20 \pm 11$ & $38 \pm 7$ & $-9 \pm 9$ \\
\hline $\mathrm{SAC}_{3}$ & $44 \pm 10$ & $18 \pm 29$ & $11 \pm 63$ & $30 \pm 22$ & $59 \pm 19$ & $2 \pm 14$ & $29 \pm 25$ & $71 \pm 1$ & $5 \pm 7$ \\
\hline$\overline{\text { SAC* }_{1}}$ & $23 \pm 17$ & $26 \pm 20$ & $50 \pm 33$ & $9 \pm 9$ & $28 \pm 8$ & $24 \pm 40$ & $45 \pm 26$ & $59 \pm 19$ & $55 \pm 37$ \\
\hline $\mathrm{SAC}_{2}$ & $26 \pm 14$ & $40 \pm 18$ & $29 \pm 18$ & $11 \pm 10$ & $14 \pm 21$ & $16 \pm 61$ & $25 \pm 17$ & $7 \pm 11$ & $60 \pm 24$ \\
\hline $\mathrm{SAC*}_{3}$ & $27 \pm 15$ & $19 \pm 16$ & $53 \pm 34$ & $12 \pm 7$ & $36 \pm 27$ & $24 \pm 65$ & $24 \pm 24$ & $18 \pm 12$ & $24 \pm 20$ \\
\hline $\mathrm{SAC}_{1}^{\mathrm{F}}$ & $42 \pm 41$ & $47 \pm 34$ & $65 \pm 14$ & $66 \pm 9$ & $74 \pm 12$ & $54 \pm 14$ & $85 \pm 9$ & $63 \pm 22$ & $65 \pm 11$ \\
\hline $\mathrm{SAC}_{2}{ }_{2}$ & $37 \pm 23$ & $41 \pm 30$ & $32 \pm 57$ & $29 \pm 37$ & $18 \pm 39$ & $29 \pm 18$ & $75 \pm 13$ & $55 \pm 30$ & $59 \pm 21$ \\
\hline $\mathrm{SAC}_{3}{ }_{3}$ & $34 \pm 41$ & $40 \pm 33$ & $42 \pm 13$ & $16 \pm 47$ & $17 \pm 41$ & $14 \pm 29$ & $32 \pm 35$ & $45 \pm 26$ & $37 \pm 40$ \\
\hline
\end{tabular}

Foram feitas cinco amostragens em cada fase. Fase l: fase de adaptação, em que os SACESHs receberam ARC diluída. Fase II: aumento da taxa de aplicação orgânica aos SACESHs em três diluições (50, 75 e 100\%). Fase III: aumento do TDH em aproximadamente duas vezes ao aplicado na fase II

Masbough et al. (2005) obtiveram remoção de 51 a $63 \%$ na DBO no tratamento de lixívia de celulose em sistemas alagados com TDH de 7 dias e DBO variando de 1.700 a $3.460 \mathrm{mg} \mathrm{L}^{-1}$. Os autores observaram maiores eficiências na remoção de poluentes naquelas células que receberam a lixívia com correção de $\mathrm{pH}(6,0)$ e adição de nutrientes (N, Pe K). Tao et al. (2006), também tratando lixívia da indústria de celulose, concluíram que $0,4 \mathrm{~kg} \mathrm{~m}^{-3} \mathrm{~d}^{-1}$ de DQO (1.000 $\mathrm{kg} \mathrm{ha}^{-1} \mathrm{~d}^{-1}$ de DQO, aproximadamente) é a $\mathrm{TCO}_{\mathrm{A}}$ máxima a ser aplicada em sistemas alagados construídos sem causar inibição da microbiota. Os autores concluíram, ainda, que com o aumento do TDH poderia ser obtido aumento na eficiência na remoção de matéria orgânica da água residuária.

Sohsalam \& Sirianuntapiboon (2008) aplicaram, em sistemas alagados construídos, $\mathrm{TCO}_{\mathrm{A}}$ de 612 a $1.213 \mathrm{~kg} \mathrm{ha}^{-1} \mathrm{~d}^{-1} \mathrm{de}$ DBO e obtiveram eficiências médias de 74 a $89 \%$ e 15 a $68 \%$, respectivamente, na remoção de $\mathrm{DBO}$ e $\mathrm{DQO}$ de águas residuárias do processamento da cana-de-açúcar. Diante dos resultados, os autores concluíram que a máxima $\mathrm{TCO}_{\mathrm{A}}$ a ser aplicada das águas residuárias do processamento da cana-deaçúcar em sistemas alagados construídos, é de $612 \mathrm{~kg} \mathrm{ha}^{-1} \mathrm{~d}^{-1}$ de DBO visto que, em maiores concentrações, houve redução na eficiência de remoção de matéria orgânica.

No tratamento de águas provenientes do processamento do couro, Calheiros et al. (2007), obtiveram entre 54 a $73 \%$ de remoção de DQO ao aplicarem $\mathrm{TCO}_{\mathrm{A}}$ entre 332 a $1.602 \mathrm{~kg} \mathrm{ha}^{-1} \mathrm{~d}^{-1}$ de DQO.

Neste trabalho se verifica que as $\mathrm{TCO}_{\mathrm{A}}$ aplicadas durante a fase III, quando se utilizou ARC diluída a $50 \%(\mathrm{v} / \mathrm{v})-\mathrm{SAC}^{\mathrm{F}}{ }_{1}$ (Tabela 2), bem como as porcentagens de remoção dos poluentes obtidas (Tabela 4), estão em conformidade com aquelas encontradas na literatura (Masbough et al., 2005; Tao et al., 2006; Calheiros et al., 2007; Sohsalam \& Sirianuntapiboon, 2008) para águas residuárias que apresentam compostos recalcitrantes; entretanto, as $\mathrm{TCO}_{\mathrm{A}}$ foram bem superiores às recomendadas para aplicação de efluentes domésticos em sistemas alagados construídos que, segundo USEPA (2000), deve variar de 60 a $133 \mathrm{~kg} \mathrm{ha}^{-1} \mathrm{~d}^{-1}$ de DBO. Além disso, foram verificadas maiores eficiências na remoção de matéria orgânica neste trabalho, quando se utilizaram, como tratamento primário, filtros anaeróbios $\left(\mathrm{SAC}^{\mathrm{F}} \mathrm{s}\right)$ (Tabela 4).

As taxas de carga de compostos fenólicos $\left(\mathrm{F}_{\mathrm{T}}\right)$ aplicadas nos SACs foram superiores àquelas aplicadas nos SAC"s e $\mathrm{SAC}^{\mathrm{F}} \mathrm{s}$, durante as fases I e II. Acredita-se que este fato seja devido à remoção desses compostos, proporcionada pela elevação do pH da ARC, que causou a sedimentação da matéria orgânica particulada e consequente remoção de $\mathrm{F}_{\mathrm{T}}$. Aktas et al. (2001) conseguiram uma eficiência entre 63 e 73\% na remoção de polifenóis, quando adicionaram cal à água residuária do processamento de oliva, a qual é rica em compostos fenólicos. Boukhoubza et al. (2009) removeram 60\% dos polifenóis da água residuária do processamento de oliva, cuja concentração inicial média era de $2.080 \mathrm{mg} \mathrm{L}^{-1}$, ao adicionarem $1 \%$ de cal, na relação peso/volume. Ao tratarem água residuária do processamento de oliva com cal, Khoufi et al. (2007) reportaram redução de cerca de 50\% da toxicidade, atribuída à remoção de compostos fenólicos.

Durante a fase II deu-se aumento nas concentrações de $\mathrm{F}_{\mathrm{T}}$, tanto afluente como efluente aos SACESHs, devido à maior concentração da ARC e à maior $\mathrm{TCO}_{\mathrm{A}}$ (Tabela 2); consequentemente, houve redução nas eficiências de remoção desta variável. Durante a fase III esta redução foi nas concentrações de $\mathrm{F}_{\mathrm{T}}$ na ARC. $\mathrm{O}$ aumento na eficiência de remoção na terceira fase nos SAC"s provavelmente se deu em função da redução na taxa de carga afluente e dos maiores valores de $\mathrm{pH}$ observados nessas unidades, os quais favoreceram a precipitação dos compostos. $\mathrm{O}$ aumento do $\mathrm{pH}$ também pode ter favorecido a remoção de $\mathrm{F}_{\mathrm{T}}$ nos $\mathrm{SAC}^{\mathrm{F}} \mathrm{s}$, apesar do aumento na taxa de carga dessas substâncias, ocorrido nesta fase; no entanto, verificou-se diminuição na eficiência de remoção de $\mathrm{F}_{\mathrm{T}}$ com o aumento da $\mathrm{TCO}_{\mathrm{A}}$ aplicada nos $\mathrm{SAC}_{2}{ }_{2}$ $\mathrm{e} \mathrm{SAC}^{\mathrm{F}}$. Grismer et al. (2003) utilizando sistemas alagados construídos no tratamento de águas residuárias da produção de vinho, obtiveram remoções de 48 a $78 \%$ de $\mathrm{F}_{\mathrm{T}}$.

Ante os resultados obtidos neste trabalho acredita-se que, entre os SACESFs avaliados, o $\mathrm{SAC}_{1}^{\mathrm{F}}$ tenha reunido as melhores condições ao desenvolvimento das espécies cultivadas (Fia et al., 2008) e ao desenvolvimento da microbiota responsável pela maior degradação da matéria orgânica. Dentre 
essas condições pode-se citar: valores de $\mathrm{pH}$ dentro da faixa considerada ideal, menores concentrações de compostos fenólicos, concentração de nutrientes e carga orgânica favorável ao processo de degradação biológica, e prétratamento em filtro anaeróbio, o qual facilitou a degradação da matéria orgânica. Além disso, acredita-se que o aumento do TDH nos SACESHs poderia aumentar ainda mais a eficiência de remoção de poluentes da ARC, principalmente para os $\mathrm{SAC}^{\mathrm{F}} \mathrm{S}$, pois é necessário um longo tempo de exposição do material orgânico da água residuária às bactérias e às enzimas por estas produzidas, para induzir e manter a degradação de polímeros tóxicos e recalcitrantes (Metcalf \& Eddy, 2003), como os compostos fenólicos.

Deve-se ressaltar que, apesar de os resultados obtidos serem promissores, o experimento foi avaliado apenas para uma safra dos frutos do cafeeiro (130 dias). Assim, mais estudos devem ser realizados para a confirmação do bom desempenho dos SACESHs quanto à remoção de matéria orgânica e compostos fenólicos da ARC.

\section{Conclusões}

1. O aumento nas taxas de carga superficial dos constituintes da ARC nos sistemas, proporcionou decréscimo na eficiência de remoção de DQO e DBO em todos os SACESHs avaliados.

2. Tempos de detenção hidráulica superiores a $100 \mathrm{~h}$ proporcionaram maiores eficiências na remoção de DQO e DBO que os de aproximadamente $60 \mathrm{~h}$, aplicados nas fases iniciais do experimento.

3. A adição de nutrientes favoreceu a remoção de matéria orgânica contida na ARC, sobretudo nos sistemas que receberam menores taxas de aplicação orgânica.

4. Entre os SACESHs, o $\mathrm{SAC}_{1}^{\mathrm{F}}$, que recebeu a menor carga orgânica apresentou desempenho mais satisfatório no que se refere à remoção de matéria orgânica e compostos fenólicos dos efluentes dos filtros anaeróbios utilizados no tratamento da ARC.

5. Na fase III a remoção de DQO no $\mathrm{SAC}^{\mathrm{F}}$, foi estatisticamente diferente $(\mathrm{p}<0,1)$ dos demais SACESHs que receberam cargas orgânicas semelhantes.

\section{LITERATURA CITADA}

Aktas, E. S.; Imre, S.; Ersoy, L. Characterization and lime treatment of olive mill wastewater. Water Research, v.35, n. 9, p.2336-2340, 2001.

APHA - American Public Health Association. Standard methods for the examination of water and wastewater. 21.ed. Washington: APHA/AWWA/WEF, 2005. [s.n.p.].

Boukhoubza, F.; Jail, A.; Korchi, F.; Idrissi, L. L.; Hannache, H.; Duarte, J. C.; Hassani, L.; Nejmeddine, A. Application of lime and calcium hypochlorite in the dephenolisation and discolouration of olive mill wastewater. Journal of Environmental Management, v.91, p.124-132, 2009.
Brasil, M. S.; Matos, A. T.; Fia, R. Eficiência e impactos ambientais do tratamento de águas residuárias da lavagem e despolpa de frutos do cafeeiro em áreas alagadas naturais. Engenharia na Agricultura, v.11, n.1-4, p.43-51, 2003.

Brasil, M. S.; Matos, A. T.; Soares; A. A.; Ferreira, P. A. Qualidade de efluente de sistemas alagados construídos, utilizados no tratamento de esgoto doméstico. Revista Brasileira de Engenharia Agrícola e Ambiental, v.9, p.133$137,2005$.

Bruno, M.; Oliveira, R. A. Tratamento anaeróbio de águas residuárias do beneficiamento de café por via úmida em reatores UASB em dois estágios. Engenharia Agrícola, v.28, n.2, p.364-377, 2008.

Calheiros, C. S. C.; Rangel, A. O. S. S.; Castro, P. M. L. Constructed wetland systems vegetated with different plants applied to the treatment of tannery wastewater. Water Research, v.41, p.1790-1798, 2007.

Dinsdale, R. M.; Hawkes, F. R.; Hawkes, D. L. The mesophilic and thermophilic anaerobic digestion of coffee waste containing coffee grounds. Water Research, v.30, n.2, p.371377, 1996.

Fia, F. R.L.; Borges, A. C.; Matos, A. T.; Duarte, I. C. S.; Fia, R.; Campos, L. C. Development of biofilm in anaerobic reactors treating wastewater from coffee grain processing. Revista Brasileira de Engenharia Agrícola e Ambiental, v.14, n.2, p.210217, 2010.

Fia, R.; Matos, A. T.; Ferreira, P. A.; Teodoro, P. E. P.; Schuery, F. C.; Luiz, F. A. R.; Desempenho agronômico da Typha sp. e Alternanthera philoxeroides Mart utilizadas no tratamento de águas residuárias da lavagem e descascamento/despolpa dos frutos do cafeeiro em sistema alagado construído. Engenharia na Agricultura, v.16, n.4, p. 436-448, 2008.

Fia, R.; Matos, A. T.; Luiz, F. A. R.; Pereira, P. A. Coeficientes de degradação da matéria orgânica de água residuária da lavagem e descascamento dos frutos do cafeeiro em condições anóxica e aeróbia. Engenharia na Agricultura, v.15, n.1, p. 45-54, 2007.

Fia, R.; Matos, A. T.; Luiz, F. A. R.; Schuery, F. C.; Teodoro, P. E. P. Desempenho de filtros anaeróbios no tratamento de águas residuárias da lavagem e descascamento/despolpa dos frutos do cafeeiro. In: Congresso Brasileiro de Engenharia Sanitária e Ambiental, 25, 2009, Recife. Anais ... Recife: ABES, 2009. CD-Rom.

Folin, O.; Ciocalteu, V. On tyrosine and tryptophane determinations in proteins. The Journal of Biological Chemistry, v.73, n.2, p.627-650, 1927.

Grismer, M. E.; Carr, M. A.; Shepherd, H. L. Evaluation of constructed wetland treatment performance for winery wastewater. Water Environmental Research, v.75, n.5, p.412421, 2003.

Khoufi, S.; Feki, F.; Sayadi, S. Detoxification of olive mill wastewater by electrocoagulation and sedimentation processes. Journal of Hazardous Materials, v. 142, p.58-67, 2007. 
Masbough, A.; Frankowski, K.; Hall, K. J.; Duff, S. J. B. The effectiveness of constructed wetland for treatment of woodwaste leachate. Ecological Engineering, v.25, p.552$566,2005$.

Matos, A. T.; Freitas, W. S.; LO Monaco, P. A. V. Eficiência de sistemas alagados construídos na remoção de poluentes de águas residuárias da suinocultura. Revista Ambiente e Água, v.5, n.2, p.119-132, 2010.

Mazzola, M.; Roston, D. M.; Valentim, M. A. A. Uso de leitos cultivados de fluxo vertical por batelada no pós-tratamento de efluente de reator anaeróbio compartimentado. Revista Brasileira de Engenharia Agrícola e Ambiental, v.9, n.2, p.276283, 2005.

Metcalf \& Eddy, Inc. Wastewater Engineering, Treatment and Reuse. 4.ed. New York: McGraw-Hill, 2003. 1819p.
Sohsalam, P.; Sirianuntapiboon, S. Feasibility of using constructed wetland treatment for molasses wastewater treatment. Bioresource Technology, v.99, n.13, p.5610-5616, 2008.

Tao, W.; Hall, K. J.; Duff, S. J. B. Performance evaluation and effects of hydraulic retention time and mass loading rate on treatment of woodwaste leachate in surface-flow constructed wetlands. Ecological Engineering, v.26, p.252265, 2006.

USEPA - United State Environmental Protection Agency. Wastewater technology fact sheet wetlands: subsurface flow. Washington: USEPA, 2000. [s.n.p.]. EPA 832-F-00-023.

Vrhovsek, D.; Kukanja, V.; Bulc, T. Constructed wetland (cw) for industrial waste water treatment. Water Research, v.30, n.10, p.2287-2292, 1996. 